\title{
Stability of de Sitter Solutions in Non-local Cosmological Models
}

\section{Emilio Elizalde}

Instituto de Ciencias del Espacio (ICE/CSIC) and Institut d'Estudis Espacials de Catalunya, Campus UAB, Facultat de Ciències, Torre C5, E-08193, Bellaterra (Barcelona), Spain

E-mail: elizaldedieec.uab.es

\section{Ekaterina O. Pozdeeva}

Skobeltsyn Institute of Nuclear Physics, Lomonosov Moscow State University,

Leninskie Gory 1, 119991, Moscow, Russia

E-mail: pozdeeva@www-hep.sinp.msu.ru

\section{Sergey Yu. Vernov*}

Skobeltsyn Institute of Nuclear Physics, Lomonosov Moscow State University, Leninskie Gory 1, 119991, Moscow, Russia

Instituto de Ciencias del Espacio (ICE/CSIC) and Institut d'Estudis Espacials de Catalunya, Campus UAB, Facultat de Ciències, Torre C5, E-08193, Bellaterra (Barcelona), Spain

E-mail: svernovdtheory.sinp.msu.ru, vernovdieec.uab.es

A non-local gravity model, which includes a function $f\left(\square^{-1} R\right)$, where $\square$ is the d'Alembert operator, is considered. For the model with an exponential $f\left(\square^{-1} R\right)$ de Sitter solutions are explored, without any restrictions on the parameters. Using Hubble-normalized variables, the stability of the de Sitter solutions is investigated, with respect to perturbations in the Bianchi I metric, in the case of zero cosmological constant, and sufficient conditions for stability are obtained.

PACS: $04.50 . \mathrm{Kd}, 95.36 .+\mathrm{x}, 98.80 .-\mathrm{k}$

The XXth International Workshop High Energy Physics and Quantum Field Theory September 24 - October 1, 2011

Sochi, Russia

\footnotetext{
*Speaker.
} 


\section{Introduction}

Modern cosmological observations allow to obtain joint constraints on cosmological parameters (see, for example [U]) and indicate that the current expansion of the universe is accelerating. The simplest model able to reproduce this late-time cosmic acceleration is general relativity with a cosmological constant. Other models involve modifications of gravity, as for instance $F(R)$ gravity, with $F(R)$ an (in principle) arbitrary function of the scalar curvature (for reviews see [■, []]).

Higher-derivative corrections to the Einstein-Hilbert action are being actively studied in the context of quantum gravity (as one of the first papers we can mention [四]). A non-local gravity theory obtained by taking into account quantum effects has been proposed in [5]. Also, the string/M-theory is usually considered as a possible theory for all fundamental interactions, including gravity. The appearance of nonlocality within string field theory is a good motivation for studying non-local cosmological models. Most of the non-local cosmological models explicitly include a function of the d'Alembert operator, $\square$, and either define a non-local modified grav-

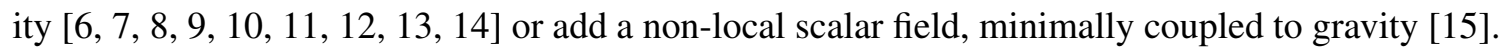

In this paper we consider a modification which includes a function of the $\square^{-1}$ operator. This modification does not assume the existence of a new dimensional parameter in the action and the ensuing non-local model has a local scalar-tensor formulation. The most currently studied

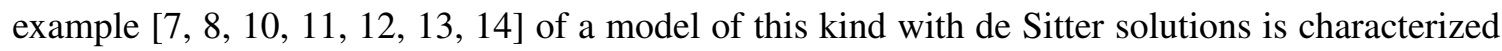
by a function $f\left(\square^{-1} R\right)=f_{0} e^{\left(\square^{-1} R\right) / \beta}$, where $f_{0}$ and $\beta$ are real parameters. It has been shown in [ $\left.\square\right]$ that a theory of this kind, being consistent with Solar System tests, may actually lead to the known universe history sequence: inflation, radiation/matter dominance and a dark epoch. Expanding universe solutions $a \sim t^{n}$ have been found in [प, [3]]. In [ए]] the ensuing cosmology at the four basic epochs: radiation dominated, matter dominated, accelerating, and a general scaling has been studied for non-local models involving, in particular, an exponential form of $f(\eta)$. An explicit mechanism to screen the cosmological constant in non-local gravity was discussed in [ए], [12, [13]].

De Sitter solutions play a very important role in cosmological models, because both inflation and the late time Universe acceleration can be described as a de Sitter solution with perturbations. A few de Sitter solutions for this model have been found in [प] and also analyzed in [ए2]. In [प4]] de Sitter solutions have been obtained without any restriction and it has been shown that the model can have de Sitter solutions only if the function $f\left(\square^{-1} R\right)$ satisfies a given second order linear differential equation. The simplest solution of this equation is an exponential function.

The Bianchi I metric can be considered as a minimal generalization of Friedmann-LemaitreRobertson-Walker (FLRW) spatially flat metric. Considering the stability of de Sitter solutions in Bianchi I metric we include anisotropic perturbations in our consideration. For the model with the exponential function $f\left(\square^{-1} R\right)$ and nonzero cosmological constant $\Lambda$ the stability of de Sitter solutions in the Bianchi I metric has been analysed in [14].

In the case $\Lambda=0$ the stability of the fixed point for the system of equations in terms of Hubblenormalized variables has been discussed in [U] and further investigated in [ [8, [4]]. In all these papers the stability of solutions has been analysed only with respect to isotropic perturbations of the initial conditions, in other words, in the FLRW metric. Here we investigate the stability of de Sitter solutions at $\Lambda=0$ in the Bianchi I metric, and show that the stability conditions, in the Bianchi I metric and in the FLRW metric, are the same. 


\section{Non-local gravitational models in the Bianchi I metric}

Consider the following action for non-local gravity

$$
S=\int d^{4} x \sqrt{-g}\left\{\frac{1}{2 \kappa^{2}}\left[R\left(1+f\left(\square^{-1} R\right)\right)-2 \Lambda\right]+\mathscr{L}_{\text {matter }}\right\}
$$

where $\kappa^{2} \equiv 8 \pi / M_{\mathrm{Pl}}{ }^{2}$, the Planck mass being $M_{\mathrm{Pl}}=1.2 \times 10^{19} \mathrm{GeV}$. The determinant of the metric tensor $g_{\mu \nu}$ is $g, \Lambda$ is the cosmological constant, $f$ is a differentiable function, and $\mathscr{L}_{\text {matter }}$ is the matter Lagrangian. We use the signature $(-,+,+,+)$.

Note that the modified gravity action (ㅁ. $(\mathbb{I})$ does not include a new dimensional parameter. This non-local model has a local scalar-tensor formulation. Introducing two scalar fields, $\eta$ and $\xi$, we can rewrite action (‥J) in the following local form:

$$
S=\int d^{4} x \sqrt{-g}\left\{\frac{1}{2 \kappa^{2}}[R(1+f(\eta)-\xi)+\xi \square \eta-2 \Lambda]+\mathscr{L}_{\text {matter }}\right\} .
$$

By varying the action ([2.2) over $\xi$, we get $\square \eta=R$. Substituting $\eta=\square^{-1} R$ into action ([2.2), one reobtains action (2.]).

Variation of action (2.2]) with respect to $\eta$ yields $\square \xi+f^{\prime}(\eta) R=0$, where the prime denotes derivative with respect to $\eta$. Varying action (2.2) with respect to the metric tensor $g_{\mu \nu}$ yields

$$
\begin{aligned}
& \frac{1}{2} g_{\mu \nu}\left[R(1+f(\eta)-\xi)-\partial_{\rho} \xi \partial^{\rho} \eta-2 \Lambda\right]-R_{\mu v}(1+f(\eta)-\xi)+ \\
+ & \frac{1}{2}\left(\partial_{\mu} \xi \partial_{\nu} \eta+\partial_{\mu} \eta \partial_{\nu} \xi\right)-\left(g_{\mu v} \square-\nabla_{\mu} \partial_{v}\right)(f(\eta)-\xi)+\kappa^{2} T_{\operatorname{matter} \mu v}=0,
\end{aligned}
$$

where $\nabla_{\mu}$ is the covariant derivative and $T_{\operatorname{matter} \mu \nu}$ the energy-momentum tensor of matter.

Let us consider the Bianchi I metric with the interval

$$
d s^{2}=-d t^{2}+a_{1}^{2}(t) d x_{1}^{2}+a_{2}^{2}(t) d x_{2}^{2}+a_{3}^{2}(t) d x_{3}^{2} .
$$

The Bianchi universe models are spatially homogeneous anisotropic cosmological models. Interpreting the solutions of the Friedmann equations as isotropic solutions in the Bianchi I metric, we include anisotropic perturbations in our consideration. A similar stability analysis has been made for cosmological models with scalar fields and phantom scalar fields in [ए]]. It is convenient to express $a_{i}$ in terms of new variables $a$ and $\beta_{i}$ (we use the notation of [ए]]):

$$
a_{i}(t)=a(t) e^{\beta_{i}(t)} .
$$

Imposing the constraint $\beta_{1}(t)+\beta_{2}(t)+\beta_{3}(t)=0$, at any $t$, one has the following relations

$$
a(t)=\left[a_{1}(t) a_{2}(t) a_{3}(t)\right]^{1 / 3}, \quad H_{i} \equiv \frac{\dot{a}_{i}}{a_{i}}=H+\dot{\beta}_{i}, \quad \text { and } \quad H \equiv \frac{\dot{a}}{a}=\frac{1}{3}\left(H_{1}+H_{2}+H_{3}\right) .
$$

In the case of the FLRW spatially flat metric we have $a_{1}=a_{2}=a_{3}=a$, all $\beta_{i}=0$, and $H$ is the Hubble parameter. Following [[1]], we introduce the shear

$$
\sigma^{2} \equiv \dot{\beta}_{1}^{2}+\dot{\beta}_{2}^{2}+\dot{\beta}_{3}^{2}
$$


In the Bianchi I metric $R=12 H^{2}+6 \dot{H}+\sigma^{2}$. The equations of motion for the scalar fields are as follows:

$$
\begin{gathered}
\ddot{\eta}=-3 H \dot{\eta}-12 H^{2}-6 \dot{H}-\sigma^{2} . \\
\ddot{\xi}=-3 H \dot{\xi}+\left(12 H^{2}+6 \dot{H}+\sigma^{2}\right) f^{\prime}(\eta),
\end{gathered}
$$

For a perfect matter fluid, we have $T_{\text {matter } 00}=\rho_{\mathrm{m}}$ and $T_{\text {matter } i j}=P_{\mathrm{m}} g_{i j}$. The equation of state is

$$
\dot{\rho}_{\mathrm{m}}=-3 H\left(P_{\mathrm{m}}+\rho_{\mathrm{m}}\right) \text {. }
$$

The Einstein equations have the form:

$$
\begin{gathered}
{\left[\frac{\sigma^{2}}{2}-3 H^{2}\right](1+\phi-\xi)+\frac{1}{2} \dot{\xi} \dot{\eta}-3 H(\dot{\phi}-\dot{\xi})+\Lambda+\kappa^{2} \rho_{\mathrm{m}}=0} \\
{\left[2 \dot{H}+3 H^{2}+\frac{\sigma^{2}}{2}-\ddot{\beta}_{j}-3 H \dot{\beta}_{j}\right](1+\phi-\xi)+\frac{1}{2} \dot{\xi} \dot{\eta}+\ddot{\phi}-\ddot{\xi}+\left(2 H-\dot{\beta}_{j}\right)(\dot{\phi}-\dot{\xi})=\Lambda-\kappa^{2} P_{\mathrm{m}}}
\end{gathered}
$$

where $\phi \equiv f(\eta)$. Summing Eqs. ([2.12) for $j=1,2,3$, we get

$$
\left[2 \dot{H}+3 H^{2}+\frac{\sigma^{2}}{2}\right](1+\phi-\xi)+\frac{1}{2} \dot{\xi} \dot{\eta}+\ddot{\phi}-\ddot{\xi}+2 H(\dot{\phi}-\dot{\xi})=\Lambda-\kappa^{2} P_{\mathrm{m}} .
$$

From equations (12.12) it is easy to get

$$
\begin{gathered}
{\left[\ddot{\beta}_{j}+3 H \dot{\beta}_{j}\right](1+\phi-\xi)+\dot{\beta}_{j}(\dot{\phi}-\dot{\xi})=0,} \\
{\left[\frac{d}{d t}\left(\sigma^{2}\right)+6 H \sigma^{2}\right](1+\phi-\xi)+2 \sigma^{2}(\dot{\phi}-\dot{\xi})=0 .}
\end{gathered}
$$

The functions $H(t), \sigma^{2}(t), \xi(t), \eta(t)$, and $\rho_{m}(t)$ can be obtained from equations ([.8) $-(\mathbb{2} .1 \mathrm{~d})$ ), (2.13) and (2.15). If $H(t)$ and the scalar fields are known, then $\beta_{j}(t)$ can be found from (2.14).

Following [12], we consider matter with a state parameter $w_{\mathrm{m}} \equiv P_{\mathrm{m}} / \rho_{\mathrm{m}}$ which is a constant not equal to -1 . Thus, Eq. (2] $)$ has the following general solution

$$
\rho_{\mathrm{m}}=\rho_{0} e^{-3\left(1+w_{\mathrm{m}}\right) H_{0} t}
$$

where $\rho_{0}$ is an arbitrary constant.

It has been shown in [14] that the model (2.2) can has de Sitter solutions for the following forms of the function $f$ :

$$
\begin{gathered}
f_{1}(\eta)=\frac{C_{2}}{4} e^{\eta / 2}+C_{3} e^{3 \eta / 2}+C_{4}-\frac{\kappa^{2} \rho_{0}}{3\left(1+3 w_{\mathrm{m}}\right) H_{0}^{2}} e^{3\left(w_{\mathrm{m}}+1\right) \eta / 4}, \quad \text { for } \quad w_{\mathrm{m}} \neq-\frac{1}{3} \\
\tilde{f}_{1}(\eta)=\frac{C_{2}}{4} e^{\eta / 2}+C_{3} e^{3 \eta / 2}+C_{4}+\frac{\kappa^{2} \rho_{0}}{4 H_{0}^{2}}\left(1-\frac{1}{3} \eta\right) e^{\eta / 2}, \quad \text { for } \quad w_{\mathrm{m}}=-\frac{1}{3}
\end{gathered}
$$

where $C_{2}, C_{3}$, and $C_{4}$ are arbitrary constants. One can see that the key ingredient in all of these functions $f_{i}(\eta)$ is an exponent function. For the models with $f(\eta)$ equal to an exponential function or a sum of exponential functions, particular de Sitter solutions have been found in [प, प2]]. In the most general form, de Sitter solutions for the case of the exponential function $f(\eta)$ have been obtained in [14]]. 


\section{De Sitter solutions and their stability}

Let us consider the action (․2.2), with

$$
f(\eta)=f_{0} e^{\eta / \beta}
$$

where $f_{0}$ and $\beta$ are real constants. This form of $f(\eta)$ is the simplest function which belongs to the set of functions described by (2.17). De Sitter solutions with a constant nonzero $H=H_{0}$ have the following expression [14]]

$$
\begin{aligned}
& \eta(t)=-4 H_{0}\left(t-t_{0}\right), \\
& \xi(t)=-\frac{3 f_{0} \beta}{3 \beta-4} e^{-\frac{4 H_{0}\left(t-t_{0}\right)}{\beta}}+\frac{c_{0}}{3 H_{0}} e^{-3 H_{0}\left(t-t_{0}\right)}-\xi_{0}, \quad \text { at } \quad \beta \neq 4 / 3, \\
& \xi(t)=-f_{0}\left(c_{0}+3 H_{0}\left(t-t_{0}\right)\right) e^{-3 H_{0}\left(t-t_{0}\right)}-\xi_{0}, \quad \text { at } \quad \beta=4 / 3,
\end{aligned}
$$

where $c_{0}$ and $t_{0}$ are arbitrary constants,

$$
\xi_{0}=-1-\frac{\Lambda}{3 H_{0}^{2}}, \quad \rho_{0}=\frac{6(\beta-2) H_{0}^{2} f_{0}}{\kappa^{2} \beta}, \quad w_{\mathrm{m}}=-1+\frac{4}{3 \beta} .
$$

The case $\beta=2$ corresponds to $\rho_{0}=0$. Thus, the model with exponential $f(\eta)$ has no de Sitter solution if we add matter with $w_{\mathrm{m}}=-1 / 3$. The type of function $f(\eta)$, which can have such solutions, is given by (2.18). The case $\beta=4 / 3$ corresponds to dark matter, because $w_{\mathrm{m}}=0$.

Using (2.8) and (2.9), we get equation (2.13]) in the form

$2\left[1+\frac{\beta-6}{\beta} \phi-\xi\right] \dot{H}=4 H\left[\frac{\phi \dot{\eta}}{\beta}-\dot{\xi}\right]-\frac{\phi \dot{\eta}^{2}}{\beta^{2}}+\frac{24}{\beta} H^{2} \phi-\dot{\xi} \dot{\eta}-\frac{4 \kappa^{2}}{3 \beta} \rho_{\mathrm{m}}-\left[1+\frac{\beta-2}{\beta} \phi-\xi\right] \sigma^{2}$.

For all $H_{0}>0$ and $\beta>0$,

$$
\phi \rightarrow 0, \quad \xi \rightarrow-\xi_{0}, \quad \text { at } \quad t \rightarrow+\infty
$$

Therefore, the coefficient of $\dot{H}$ in (B. (B) tends to $\Lambda /\left(3 H_{0}^{2}\right)$. In the case of nonzero $\Lambda$, the stability of de Sitter solutions at late times can be analysed without using of the Hubble-normalized variables. It has been found in [14] that for $H_{0}>0$ and $\beta>0$, the de Sitter solutions are stable with respect to fluctuations of the initial conditions in the Bianchi I metric at any nonzero value of $\Lambda$.

Here we consider the stability of de Sitter solutions with respect to fluctuations of the initial conditions in the Bianchi I metric, in the case $\Lambda=0$. To analyze the stability of the de Sitter solutions at $\Lambda=0$, we transform the system of equations using the Hubble-normalized variables

$$
X=-\frac{\dot{\eta}}{4 H}, \quad W=\frac{\dot{\xi}}{6 H f}, \quad Y=\frac{1-\xi}{3 f}, \quad Z=\frac{\kappa^{2} \rho_{m}}{3 H^{2} f}, \quad K=\frac{\sigma^{2}}{2 H^{2}}
$$

and the independent variable, $N$,

$$
\frac{d}{d N} \equiv a \frac{d}{d a}=\frac{1}{H} \frac{d}{d t}
$$




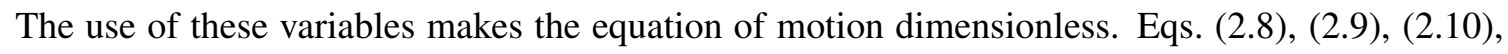
and Eq. ([2.15) are equivalent to the following ones, in terms of the new variables,

$$
\begin{aligned}
\frac{d X}{d N} & =3(1-X)+\frac{1}{H}\left(\frac{3}{2}-X\right) \frac{d H}{d N}+\frac{K}{2} \\
\frac{d W}{d N} & =\frac{2}{\beta}(1+2 W X)-3 W+\frac{1}{H}\left(\frac{1}{\beta}-W\right) \frac{d H}{d N}+\frac{K}{3 \beta}, \\
\frac{d Z}{d N} & =\frac{4}{\beta}(X-1) Z-2 \frac{Z}{H} \frac{d H}{d N} \\
\left(\frac{d K}{d N}\right. & \left.+\frac{2 K}{H} \frac{d H}{d N}+6 K\right)(3 Y+1)=4 K\left(\frac{2 X}{\beta}+3 W\right) .
\end{aligned}
$$

To get the full system of the first order differential equations we need to get one for $\frac{d H}{d N}$ and to eliminate $Y$. To do this, we use Eq. (대), which can be written in terms of the new variables as

$$
Y=-\frac{1}{3}+\frac{2 \beta(2 X-3) W-4 X-\beta Z}{\beta(K-3)} .
$$

Differentiating (B.14), substituting (B.10) $-($ (B.13) $)$, and using

$$
\frac{d Y}{d N}=2\left(\frac{2 X Y}{\beta}-W\right)=\frac{4 X}{3 \beta^{2}(3-K)}(\beta(K-3)+6 \beta(3-2 X) W+12 X+3 \beta Z)-2 W,
$$

one gets

$$
\begin{aligned}
& (2(2 X-3)(\beta W-1)-\beta Z-2 K) \frac{1}{H} \frac{d H}{d N}=\frac{8(3-K) X^{2}}{3 \beta}+ \\
& +\frac{4}{3}(6-9 \beta W+K) X+2 Z+12(\beta W-1)+\left(2-\frac{2}{3} Z+(2 W+Z) \beta\right) K+\frac{2}{3} K^{2},
\end{aligned}
$$

In terms of the new variables system (B.JU) $-(B .53)$, (B.J6) has the following fixed point

$$
H=H_{0}, \quad X_{0}=1, \quad Z_{0}=\frac{2(\beta-2)}{\beta}, \quad W_{0}=\frac{2}{3 \beta-4}, \quad K_{0}=0,
$$

which corresponds to de Sitter solution for $\beta \neq 4 / 3$, with $c_{0}=0$. In the case of an arbitrary $c_{0}$, for the de Sitter solution, we get

$$
W=\frac{2}{3 \beta-4}-\frac{c_{0}}{6 H_{0} f_{0}} e^{-(3-4 / \beta)\left(N-N_{0}\right)},
$$

where $N_{0}=H_{0} t_{0}$. The function $W$ tends to infinity at large $N$ for $\beta<4 / 3$ and $\lim _{N \rightarrow \infty} W=W_{0}$ at $\beta>4 / 3$. So, the fixed point can be stable only at $\beta>4 / 3$. Under this condition all de Sitter solutions tend to a fixed point, what means that, for any $\varepsilon>0$, there exists a number, $N_{1}$, such that the de Sitter solution is in the $\varepsilon / 2$ neighborhood of the fixed point, for all $N>N_{1}$. Therefore, the stability of the fixed point guarantees the stability of all de Sitter solutions.

For $\beta=4 / 3$ the function $W$, corresponding to de Sitter solutions, depends on $N$ for any value of parameters. Thus, this choice of dimensionless variable is not suitable to analyse stability of the de Sitter solutions for $\beta=4 / 3$. Here we will deal with the case $\beta \neq 4 / 3$, only. 
Let us consider perturbations in the neighborhood of (B.T7):

$$
X=1+\varepsilon x_{1}, \quad Z=Z_{0}\left(1+\varepsilon z_{1}\right), \quad W=W_{0}\left(1+\varepsilon w_{1}\right), \quad H=H_{0}\left(1+\varepsilon h_{1}\right), \quad K=\varepsilon k_{1} .
$$

To first order in $\varepsilon$, after some work we obtain the system of linear equations:

$$
\begin{gathered}
\frac{d x_{1}}{d N}=-3 x_{1}+\frac{1}{2} \frac{d h_{1}}{d N}+\frac{1}{2} k_{1}, \quad \frac{d z_{1}}{d N}=\frac{4}{\beta} x_{1}-2 \frac{d h_{1}}{d N}, \\
\frac{d w_{1}}{d N}=\frac{4}{\beta} x_{1}+\frac{\beta-4}{2 \beta} \frac{d h_{1}}{d N}+\left(\frac{4}{\beta}-3\right) w_{1}+\frac{3 \beta-4}{6 \beta} k_{1}, \\
\frac{d h_{1}}{d N}=\frac{8(4-\beta)}{\beta\left(3 \beta^{2}-11 \beta+12\right)} x_{1}-\frac{2(3 \beta-4)(\beta-2)}{\beta\left(3 \beta^{2}-11 \beta+12\right)} z_{1}-\frac{3 \beta^{2}-5 \beta+4}{3 \beta^{2}-11 \beta+12} k_{1}, \\
\frac{d k_{1}}{d N}=\left(\frac{8}{\beta}-6\right) k_{1} .
\end{gathered}
$$

Solving (3.23), we get

$$
k_{1}(N)=b_{1} e^{-(6-8 / \beta) N},
$$

where $b_{1}$ is an arbitrary constant and $k_{1}$ tends to zero for $N \rightarrow \infty$, if and only if $\beta>4 / 3$.

Substituting $k_{1}$ and (B.22) into (B.2U) , we get a system of two inhomogeneous differential equations. As known, the general solution of this system is a sum of the general solution of the corresponding homogeneous system and a particular solution of inhomogeneous one. The homogeneous system corresponds to the FLRW metric (the case $K=0$ ) and those general solution, which has been obtained in [14]], is bounded and tends to zero for $N \rightarrow \infty$, if $4 / 3<\beta \leqslant 2$. For any $\beta$ from this interval a particular solution of the inhomogeneous system tends to zero as well, because $k_{1}$ tends to zero at $\beta>4 / 3$. Therefore, the perturbations $x_{1}$ and $z_{1}$ decrease provided $4 / 3<\beta<2$. Substituting $x_{1}(N)$ and $z_{1}(N)$ into Eqs. (B.27) and (B.22) we get that $h_{1}(N)$ and $w_{1}(N)$ decrease as well. Note that $h_{1}(N)$ has a part, $H_{1}$, which does not depend on $N$ and, therefore, it can be considered as part of $H_{0}$. This result corresponds to the fact that, for $\Lambda=0$, the value of $H_{0}$ can be selected arbitrarily; thus, one can choose $\tilde{H}_{0}=H_{0}+H_{1}$ instead of $H_{0}$. We can summarize the above saying that the de Sitter solutions are stable with respect to perturbations of the Bianchi I metric, in the case $4 / 3<\beta \leqslant 2$. If $f_{0}>0$, then the stable de Sitter solution corresponds to $\rho_{0} \leqslant 0$.

\section{Conclusions}

We have here investigated de Sitter solutions in the non-local gravity model described by the action ([2.]) (see [ [ []]). We have used the local formulation of the model ([2.2), which includes two scalar fields. We have specifically considered the case of the exponential function $f(\eta)$, which is the simplest and most studied case, corresponding to the model (․2.), which admits de Sitter solutions.

In [14]] we have discussed the stability of de Sitter solutions in the Bianchi I metrics and obtained that, for $H_{0}>0$ and $\beta>0$, de Sitter solutions are stable, for all nonzero values of $\Lambda$. Here we have proved that in the case $\Lambda=0$ de Sitter solutions are stable for $H_{0}>0$ and $4 / 3<$ $\beta \leqslant 2$. Thus, our conclusion is that de Sitter solutions, which are stable with respect to isotropic perturbations, are also stable with respect to anisotropic perturbations of the Bianchi I metric. 
The authors thank Sergei D. Odintsov for useful discussions. E.E. was supported in part by MICINN (Spain), projects FIS2006-02842 and FIS2010-15640, by the CPAN Consolider Ingenio Project, and by AGAUR (Generalitat de Catalunya), contract 2009SGR-994. E.P. and S.V. are supported in part by the RFBR grant 11-01-00894, E.P. also by a state contract of the Russian Ministry of Education and Science 14.740.12.0846, and S.V. by a grant of the Russian Ministry of Education and Science NSh-4142.2010.2 and by CPAN10-PD12 (ICE, Barcelona, Spain).

\section{References}

[1] M. Kilbinger et al., Dark energy constraints and correlations with systematics from CFHTLS weak lensing, SNLS supernovae Ia and WMAP5, Astron. Astrophys. 497 (2009) 677-688 [arXiv:0810.5129]

[2] S. Nojiri and S.D. Odintsov, Unified cosmic history in modified gravity: from $F(R)$ theory to Lorentz non-invariant models, Phys. Rept. 505 (2011) 59-144 [arXiv: 1011.0544 ]

[3] S. Capozziello and V. Faraoni, Beyond Einstein Gravity: A Survey of Gravitational Theories for Cosmology and Astrophysics, Fund. Theor. Phys. 170, Springer, New York, 2011

[4] K.S. Stelle, Renormalization of Higher Derivative Quantum Gravity, Phys. Rev. D 16 (1977) 953

[5] S. Deser and R.P. Woodard, Nonlocal Cosmology, Phys. Rev. Lett. 99 (2007) 111301 [arXiv:0706.2151]

[6] T. Biswas, A. Mazumdar, and W. Siegel, Bouncing Universes in String-inspired Gravity, JCAP 0603 (2006) 009 [hep-th/0508194];

S. Capozziello, E. Elizalde, S. Nojiri, and S.D. Odintsov, Accelerating cosmologies from non-local higher-derivative gravity, Phys. Lett. B 671 (2009) 193-198 [arXiv: 0809.1535 ];

S. Nesseris and A. Mazumdar, Newton's constant in $f\left(R, R_{\mu v} R^{\mu v}, \square R\right)$ theories of gravity and constraints from BBN, Phys. Rev. D 79 (2009) 104006 [arXiv: 0902.1185 ];

C. Deffayet and R.P. Woodard, Reconstructing the Distortion Function for Nonlocal Cosmology, JCAP 0908 (2009) 023 [arXiv: 0904 .0961];

G. Cognola, E. Elizalde, S. Nojiri, S.D. Odintsov, and S. Zerbini, One-loop effective action for non-local modified Gauss-Bonnet gravity in de Sitter space, Eur. Phys. J. C 64 (2009) 483-494 [arXiv:0905.0543];

G. Calcagni and G. Nardelli, Nonlocal gravity and the diffusion equation, Phys. Rev. D 82 (2010) 123518 [arXiv: 1004.5144 ];

T. Biswas, T. Koivisto, and A. Mazumdar, Towards a resolution of the cosmological singularity in non-local higher derivative theories of gravity, JCAP 1011 (2010) 008 [arXiv: 1005 . 0590];

A.O. Barvinsky, Nonlocal gravity and its cosmological manifestations, arXiv: 1107.1463

[7] S. Nojiri and S.D. Odintsov, Modified non-local-F(R) gravity as the key for the inflation and dark energy, Phys. Lett. B 659 (2008) 821 [arXiv: 0708 .0924]

[8] S. Jhingan, S. Nojiri, S.D. Odintsov, M. Sami, I. Thongkool, and S. Zerbini, Phantom and non-phantom dark energy: The cosmological relevance of non-locally corrected gravity, Phys. Lett. B 663 (2008) 424-428 [arXiv: 0803 .2613]

[9] K.A. Bronnikov and E. Elizalde, Spherical systems in models of nonlocally corrected gravity, Phys. Rev. D 81 (2010) 044032 [arXiv: 0910.3929 ]; J. Kluson, Non-Local Gravity from Hamiltonian Point of View, JHEP 1109 (2011) 001 [arXiv:1105.6056] 
[10] T.S. Koivisto, Dynamics of Nonlocal Cosmology, Phys. Rev. D 77 (2008) 123513 [arXiv:0803.3399]

[11] S. Nojiri, S.D. Odintsov, M. Sasaki and Y.l. Zhang, Screening of cosmological constant in non-local gravity, Phys. Lett. B 696 (2011) 278 [arXiv: 1010 . 5375]

[12] K. Bamba, Sh. Nojiri, S.D. Odintsov, and M. Sasaki, Screening of cosmological constant for De Sitter Universe in non-local gravity, phantom-divide crossing and finite-time future singularities, arXiv:1104.2692

[13] Y.1. Zhang and M. Sasaki, Screening of cosmological constant in non-local cosmology, Int. J. Mod. Phys. D 21 (2012) 1250006 [arXiv:1108.2112]

[14] E. Elizalde, E.O. Pozdeeva, S.Yu. Vernov, De Sitter Universe in Non-local Gravity, Phys. Rev. D 2012, to be publishes, arXiv: 1110.5806

[15] G. Calcagni, Cosmological tachyon from cubic string field theory, JHEP 0605 (2006) 012 [hep-th/0512259];

A.S. Koshelev, Non-local SFT Tachyon and Cosmology, JHEP 0704 (2007) 029 [hep-th/0701103];

I.Ya. Aref'eva, L.V. Joukovskaya, and S.Yu. Vernov, Bouncing and accelerating solutions in nonlocal stringy models, JHEP 0707 (2007) 087 [hep-th/ 0701184 ];

I.Ya. Aref'eva and I.V. Volovich, Quantization of the Riemann Zeta-Function and Cosmology, Int. J. of Geom. Meth. Mod. Phys. 4 (2007) 881-895 [hep-th/ 0701284 ];

G. Calcagni, M. Montobbio, and G. Nardelli, A route to nonlocal cosmology, Phys. Rev. D 76 (2007) 126001 [arXiv:0705.3043];

N. Barnaby, T. Biswas, and J.M. Cline, p-adic Inflation, JHEP 0704 (2007) 056 [hep-th/0612230];

I.Ya. Aref'eva, L.V. Joukovskaya, and S.Yu. Vernov, Dynamics in nonlocal linear models in the Friedmann-Robertson-Walker metric, J. Phys. A: Math. Theor. 41 (2008) 304003 [arXiv:0711.1364];

D.J. Mulryne and N.J. Nunes, Diffusing non-local inflation: Solving the field equations as an initial value problem, Phys. Rev. D 78 (2008) 063519 [arXiv: 0805 . 044 9];

G. Calcagni, M. Montobbio, and G. Nardelli, Localization of nonlocal theories, Phys. Lett. B 662 (2008) 285-289 [arXiv:0712 .2237];

L.V. Joukovskaya, Dynamics in nonlocal cosmological models derived from string field theory Phys. Rev. D 76 (2007) 105007 [arXiv: 0707 . 1545];

N. Barnaby and N. Kamran, Dynamics with Infinitely Many Derivatives: The Initial Value Problem, JHEP 0802 (2008) 008 [arXiv: 0709 . 3968];

$\mathrm{S}$.Yu. Vernov, Localization of nonlocal cosmological models with quadratic potentials in the case of double roots, Class. Quant. Grav. 27 (2010) 035006 [arXiv:0907.0468];

S.Yu. Vernov, Localization of the SFT inspired Nonlocal Linear Models and Exact Solutions, Phys. Part. Nucl. Lett. 8 (2011) 310-320 [arXiv: 1005 . 0372];

A.S. Koshelev and S.Yu. Vernov, Analysis of scalar perturbations in cosmological models with a non-local scalar field, Class. Quant. Grav. 28 (2011) 085019 [arXiv: 1009 . 0746]

[16] I.Ya. Aref'eva, N.V. Bulatov, L.V. Joukovskaya, S.Yu. Vernov, Null Energy Condition Violation and Classical Stability in the Bianchi I Metric, Phys. Rev. D 80 (2009) 083532 [arXiv: 0903.5264 ]; I.Ya. Aref'eva, N.V. Bulatov, and S.Yu. Vernov, Stable Exact Solutions in Cosmological Models with Two Scalar Fields, Theor. Math. Phys. 163 (2010) 788-803 [arXiv:0911. 5105]

[17] T.S. Pereira, C. Pitrou, and J.-Ph. Uzan, Theory of cosmological perturbations in an anisotropic universe, JCAP 0709 (2007) 006 [arXiv: 0707 . 0736] 